\title{
Diameter pinching in almost positive Ricci curvature
}

\author{
Erwann Aubry
}

\begin{abstract}
In this paper we prove a diameter sphere theorem and its corresponding $\lambda_{1}$ sphere theorem under $L^{p}$ control of the curvature. They are generalizations of some results due to S. Ilias [8].
\end{abstract}

Mathematics Subject Classification (2000). 53C20.

Keywords. Ricci curvature, comparison theorems, integral bounds on the curvature, sphere theorems.

\section{Introduction}

Let $\left(M^{n}, g\right)$ be a complete manifold with Ricci curvature Ric $\geq n-1$. Then $\left(M^{n}, g\right)$ satisfies the following classical results (the proofs can be found in [13] for instance):

- $\operatorname{Diam}\left(M^{n}, g\right) \leq \pi$ (S. Myers) with equality iff $\left(M^{n}, g\right)=\left(\mathbb{S}^{n}\right.$, can $)$ (S. Cheng),

- $\lambda_{1}\left(M^{n}, g\right) \geq n$ (A. Lichnerowicz) with equality iff $\left(M^{n}, g\right)=\left(\mathbb{S}^{n}\right.$, can $)$ (M. Obata),

where Diam is the diameter and $\lambda_{1}$ is the first positive eigenvalue.

Studying the properties of the sphere kept by manifolds with Ric $\geq n-1$ and almost extremal diameter or $\lambda_{1}$, S. Ilias proved in [8] the following results:

Theorem 1.1 (S. Ilias). For any $A>0$, there exists $\epsilon(A, n)>0$ such that any n-manifold with Ric $\geq n-1$, sectional curvature $\sigma \leq A$ and $\lambda_{1} \leq n+\epsilon$ is homeomorphic to $\mathbb{S}^{n}$.

Theorem 1.2 (S. Ilias). For any $A>0$, there exists $\epsilon(A, n)>0$ such that any $n$-manifold with Ric $\geq n-1, \sigma \leq A$ and $\operatorname{Diam}(M) \geq \pi-\epsilon$ is homeomorphic to $\mathbb{S}^{n}$.

Remark 1.3. C. Croke proves in [7] that for $n$-manifolds with Ric $\geq n-1, \lambda_{1}(M)$ close to $n$ implies Diam $(M)$ close to $\pi$. The converse is proved in [8] (using a spectral inequality due to $\mathrm{S}$. Cheng [6]). 
Remark 1.4. For $n \geq 4$, M. Anderson [1] and Y. Otsu [10] construct sequences of complete metrics $g_{i}$ with $\operatorname{Ric}\left(g_{i}\right) \geq n-1, \lambda_{1}\left(g_{i}\right) \rightarrow n$ and $\operatorname{Diam}\left(g_{i}\right) \rightarrow \pi$ on manifolds that are not homotope to $\mathbb{S}^{n}$ (more precisely, Otsu shows that if $n \geq 5$, these manifolds can have infinitely many different fundamental groups).

Remark 1.5. The two results of S. Ilias have been improved by G. Perelman in [11], where the assumption $\sigma \leq A$ is replaced by $\sigma \geq-A$ (note that under the Ilias's assumptions $\sigma \leq A$ and Ric $\geq n-1$ we have $|\sigma| \leq(n-2) A)$.

Subsequently, we denote $\operatorname{Ric}(x)$ the lowest eigenvalue of the Ricci tensor and $\bar{\sigma}(x)$ the maximal sectional curvature at $x$. In [4], we prove the following generalization of the theorems of Myers and Lichnerowicz:

Theorem 1.6. For any $p>n / 2$, there exists $C(p, n)$ such that if $\left(M^{n}, g\right)$ is a complete manifold with $\int_{M}(\underline{\mathrm{Ric}}-(n-1))_{-}^{p}<\frac{\mathrm{Vol} M}{C(p, n)}$, then $M$ is compact, has finite fundamental group and satisfies

$$
\begin{gathered}
\operatorname{Diam}(M) \leq \pi\left[1+C(p, n)\left(\frac{\rho_{p}}{\operatorname{Vol} M}\right)^{\frac{1}{10}}\right], \\
\lambda_{1}(M) \geq n\left[1-C(p, n)\left(\frac{\rho_{p}}{\operatorname{Vol} M}\right)^{\frac{1}{p}}\right],
\end{gathered}
$$

where $\rho_{p}=\int_{M}(\underline{\operatorname{Ric}}-(n-1))_{-}^{p}$ and $x_{-}=\max (0,-x)$.

Remark 1.7. It follows from [4] that the constant $C(p, n)$ is computable, that if $\int_{M}(\underline{\mathrm{Ric}}-(n-1))_{-}^{p}$ is finite (for $\left.p>n / 2\right)$, then Vol $M$ is finite, and that we cannot bound the diameter or the first non zero eigenvalue under the assumption $\rho_{p} \leq \frac{1}{C(p, n)}$ or $\rho_{\frac{n}{2}}$ small (see [4]).

In this paper we prove the following extensions of Ilias's stability results.

Theorem 1.8. Let $n \geq 2$ be an integer, $A>0$ and $p>n$ be some reals. There exists a positive constant $C(p, n, A)$ such that any complete $n$-manifold which satisfies

and

$$
\int_{M}(\underline{\operatorname{Ric}}-(n-1))_{-}^{p}<C(p, n, A) \operatorname{Vol} M, \quad \int_{M} \bar{\sigma}_{+}^{p}<A \operatorname{Vol} M
$$

$$
\operatorname{Diam}(M) \geq \pi(1-C(p, n, A))
$$

is homeomorphic to $\mathbb{S}^{n}$ (where $x_{+}=\max (0, x)$ ).

Theorem 1.9. Let $n \geq 2$ be an integer, $A>0$ and $p>n$ be some reals. There exists a positive constant $C(p, n, A)$ such that any complete $n$-manifold which satisfies

$$
\int_{M}(\underline{\operatorname{Ric}}-(n-1))_{-}^{p}<C(p, n, A) \operatorname{Vol} M, \quad \int_{M} \bar{\sigma}_{+}^{p}<A \operatorname{Vol} M
$$


and

$$
\lambda_{1}(M) \leq n(1+C(p, n, A))
$$

is homeomorphic to $\mathbb{S}^{n}$.

Remark 1.10. By the Hölder inequality, the two curvature assumptions of Theorem 1.9 can be replaced by

$$
\int_{M}(\underline{\operatorname{Ric}}-(n-1))_{-}<C(p, n, A) \operatorname{Vol} M \text { and } \int_{M} \sigma^{p}<A \operatorname{Vol} M,
$$

where $\sigma(x)$ is an upper bound for the absolute value of the sectional curvatures at $x$.

\section{Comparison results in almost positive Ricci curvature}

Subsequently we denote $B(x, r)$ (resp. $S(x, r))$ the geodesic ball (resp. sphere) with center $x$ and radius $r$ and $L_{k}(r)$ (resp. $A_{k}(r)$ ) the volume of a geodesic sphere (resp. ball) of radius $r$ in $\left(\mathbb{S}^{n}, \frac{1}{k} g\right)$. Besides Theorem 1.6, we will need the following comparison results for manifolds of almost positive Ricci curvature (see [4] for a proof).

Proposition 2.1. For any $n \geq 2$ and $p>n / 2(p \geq 1$ if $n=2)$ there exists a constant $C(p, n)$ such that for any complete Riemannian $n$-manifold $\left(M^{n}, g\right)$ with $\eta^{10}=\frac{\rho_{p}}{\operatorname{Vol} M} \leq \frac{1}{C(p, n)}$, we have

$$
\begin{gathered}
\left(\frac{\operatorname{Vol}_{n-1} S(x, R)}{L_{1-\eta}(R)}\right)^{\frac{1}{2 p-1}}-\left(\frac{\operatorname{Vol}_{n-1} S(x, r)}{L_{1-\eta}(r)}\right)^{\frac{1}{2 p-1}} \leq C(p, n) \eta^{2}(R-r)^{\frac{2 p-n}{2 p-1}} \\
\frac{\operatorname{Vol} B(x, r)}{\operatorname{Vol} B(x, R)} \geq(1-C(p, n) \eta) \frac{A_{1}(r)}{A_{1}(R)} \\
\operatorname{Vol}_{n-1} S(x, R) \leq\left(1+\eta^{2}\right) L_{1-\eta}(R) \\
\quad \operatorname{Vol} B(x, R) \leq(1+\eta) A_{1}(R)
\end{gathered}
$$

for all $x \in M$ and all radii $0 \leq r \leq R$.

For any $n \geq 2$ and $p>n / 2$ there exists a constant $C(p, n)$ such that if $\left(M^{n}, g\right)$ is a complete n-manifold with $\bar{\rho}_{p} \leq \frac{1}{C(p, n)}$, then $\|u\|_{\frac{2 n}{n-2}} \leq \operatorname{Diam}(M) C(p, n)\|d u\|_{2}+$ $\|u\|_{2}$, for any $u \in H^{1,2}(M)$. In the casen $=2$, we have $\|u\|_{4} \leq \operatorname{Diam}(M) C\|d u\|_{2}+$ $\|u\|_{2}$ if $\bar{\rho}_{1} \leq \frac{1}{C}$.

Similar estimates are proved in [12] under the assumption that $M$ is compact and $\operatorname{Diam}(M)^{2 p} \frac{\rho_{p}}{\operatorname{Vol} M} \leq \frac{1}{C(p, n)}$. 


\section{Theorem 1.9 implies Theorem 1.8}

Proposition 3.1. Let $n \geq 2$ and $p>n / 2$. There exists $C(p, n)>0$ such that if $\left(M^{n}, g\right)$ is a complete $n$-manifold with $\eta^{10}=\bar{\rho}_{p} \leq \frac{1}{C(p, n)}$ and $\operatorname{Diam}(M) \geq$ $\pi-\frac{1}{C(p, n)}$, then we have

$$
\lambda_{1}(M) \leq n+C(p, n)\left[\eta+(\operatorname{Diam}(M)-\pi)_{-}\right] .
$$

The main tool to prove this proposition is the following lemma:

Lemma 3.2. Let $n \geq 2$ and $p>n / 2(p \geq 1$ if $n=2)$ and $\bar{x}_{0} \in \mathbb{S}^{n}$. There exists a constant $C(p, n)$ such that if $\left(M^{n}, g\right)$ is a complete $n$-manifold with $\eta^{10}=\bar{\rho}_{p} \leq$ $\frac{1}{C(p, n)}$, then there exists $x_{0} \in M$ such that for any $C^{1}$-function $u:[0,2 \pi] \rightarrow \mathbb{R}$ we have

$$
\begin{aligned}
& \left|\frac{1}{\operatorname{Vol} M} \int_{M} u \circ d_{M}\left(x_{0}, .\right) d v_{g}-\frac{1}{\operatorname{Vol} \mathbb{S}^{n}} \int_{\mathbb{S}^{n}} u \circ d_{\mathbb{S}^{n}}\left(\bar{x}_{0}, .\right) d v_{\mathbb{S}^{n}}\right| \\
& \quad \leq\left\|u^{\prime}\right\|_{\infty} C(p, n)\left[\eta+(\operatorname{Diam}(M)-\pi)_{-}\right] .
\end{aligned}
$$

Proof. Let $\left(x_{0}, y_{0}\right) \in M^{2}$ such that $d=\operatorname{Diam}(M)=d\left(x_{0}, y_{0}\right)$. The functions $A$, $L, A_{1}$ and $L_{1}$ are defined in Proposition 2.1 and prolonged by 0 to $\mathbb{R}$ (note that the diameter of $M$ can be greater than $\pi)$. The function $r \rightarrow u(r) A(r)$ is continuous and has right differential on $\mathbb{R}$ equal to $u^{\prime} A+u L$. We infer the equalities

$$
\begin{aligned}
u(d) \operatorname{Vol} M & =\int_{0}^{d} u(r) L(r) d r+\int_{0}^{d} u^{\prime}(r) A(r) d r, \\
u(\pi) \operatorname{Vol} \mathbb{S}^{n} & =\int_{0}^{\pi} u(r) L_{1}(r) d r+\int_{0}^{\pi} u^{\prime}(r) A_{1}(r) d r
\end{aligned}
$$

which imply

$$
\begin{aligned}
\left|\frac{1}{\operatorname{Vol} M} \int_{M} u \circ d_{M}\left(x_{0}, x\right) d v_{g}-\frac{1}{\operatorname{Vol} \mathbb{S}^{n}} \int_{\mathbb{S}^{n}} u \circ d_{\mathbb{S}^{n}}\left(\bar{x}_{0}, x\right) d v_{\mathbb{S}^{n}}\right| \\
\quad=\left|\int_{0}^{d} \frac{u(r) L(r)}{\operatorname{Vol} M} d r-\int_{0}^{\pi} \frac{u(r) L_{1}(r)}{\operatorname{Vol} \mathbb{S}^{n}}\right| \\
=\left|u(d)-u(\pi)+\int_{0}^{\pi} \frac{u^{\prime} A_{1}}{\operatorname{Vol} \mathbb{S}^{n}}-\int_{0}^{d} \frac{u^{\prime} A}{\operatorname{Vol} M}\right| \\
=\left|\int_{0}^{d} u^{\prime}\left(\frac{A_{1}}{\operatorname{Vol} \mathbb{S}^{n}}-\frac{A}{\operatorname{Vol} M}\right)+\int_{d}^{\pi} u^{\prime}\left(\frac{A_{1}}{\operatorname{Vol} \mathbb{S}^{n}}-1\right)\right| \\
\leq\left\|u^{\prime}\right\|_{\infty}\left(\int_{0}^{d}\left|\frac{A_{1}}{\operatorname{Vol} \mathbb{S}^{n}}-\frac{A}{\operatorname{Vol} M}\right| d r+|\pi-d|\right) .
\end{aligned}
$$


By Proposition 2.1 we have, for all $r \leq d$ :

$$
\begin{aligned}
(1-C(p, n) \eta) \frac{A_{1}(r)}{\operatorname{Vol} \mathbb{S}^{n}} & \leq \frac{A(r)}{\operatorname{Vol} M} \leq 1-\frac{\operatorname{Vol} B\left(y_{0}, d-r\right)}{\operatorname{Vol} M} \\
& \leq 1-(1-C(p, n) \eta) \frac{A_{1}(d-r)}{\operatorname{Vol} \mathbb{S}^{n}} \\
& \leq \frac{A_{1}(r+\pi-d)}{\operatorname{Vol} \mathbb{S}^{n}}+C(p, n) \eta .
\end{aligned}
$$

Hence $\left|\frac{A(r)}{\operatorname{Vol} M}-\frac{A_{1}(r)}{\operatorname{Vol} \mathbb{S}^{n}}\right| \leq C(p, n) \eta+\frac{\left(A_{1}(r)-A_{1}(r+\pi-d)\right)_{-}}{\operatorname{Vol}^{n}}$. An easy computation gives $\left\|\frac{\left(A_{1}(\cdot)-A_{1}(\cdot+h)\right)_{-}}{\operatorname{Vol} \mathbb{S}^{n}}\right\|_{\infty} \leq C(n)(-h)_{-}$, and by Proposition 2.1 we get:

$$
\begin{aligned}
& \left|\frac{1}{\operatorname{Vol} M} \int_{M} u \circ d_{M}\left(x_{0}, x\right) d v_{g}-\frac{1}{\operatorname{Vol} \mathbb{S}^{n}} \int_{\mathbb{S}^{n}} u \circ d_{\mathbb{S}^{n}}\left(\bar{x}_{0}, x\right) d v_{\mathbb{S}^{n}}\right| \\
& \quad \leq\left\|u^{\prime}\right\|_{\infty} C(p, n)\left[\eta+(d-\pi)_{-}\right] .
\end{aligned}
$$

We now finish the proof of Proposition 3.1.

Proof. Lemma 3.2 applied to $u=\sin ^{2}, u=\cos ^{2}$ and $u=\cos$ gives

$$
\begin{aligned}
\left|\int_{M} \frac{\sin ^{2} d_{M}\left(x_{0}, .\right)}{\operatorname{Vol} M}-\int_{\mathbb{S}^{n}} \frac{\sin ^{2} d_{\mathbb{S}^{n}}\left(\bar{x}_{0}, .\right)}{\operatorname{Vol} \mathbb{S}^{n}}\right| & \leq C(p, n)\left(\eta+(d-\pi)_{-}\right) \leq 1, \\
\left|\int_{M} \frac{\cos ^{2} d_{M}\left(x_{0}, .\right)}{\operatorname{Vol} M}-\int_{\mathbb{S}^{n}} \frac{\cos ^{2} d_{\mathbb{S}^{n}}\left(\bar{x}_{0}, .\right)}{\operatorname{Vol} \mathbb{S}^{n}}\right| & \leq C(p, n)\left(\eta+(d-\pi)_{-}\right) \leq 1, \\
\left|\int_{M} \frac{\cos d_{M}\left(x_{0}, .\right)}{\operatorname{Vol} M}-\int_{\mathbb{S}^{n}} \frac{\cos d_{\mathbb{S}^{n}}\left(\bar{x}_{0}, .\right)}{\operatorname{Vol} \mathbb{S}^{n}}\right| & \leq C(p, n)\left(\eta+(d-\pi)_{-}\right) \leq 1 .
\end{aligned}
$$

Hence, if we set $f=\cos d_{M}\left(x_{0},.\right)$, we get

$$
\begin{aligned}
\left|\|\nabla f\|_{2}^{2}-\frac{n}{n+1}\right| & \leq C(p, n)\left(\eta+(d-\pi)_{-}\right) \leq 1, \\
\left|\|f\|_{2}^{2}-\frac{1}{n+1}\right| & \leq C(p, n)\left(\eta+(d-\pi)_{-}\right) \leq 1, \\
\left|\frac{1}{\operatorname{Vol} M} \int_{M} f\right| & \leq C(p, n)\left(\eta+(d-\pi)_{-}\right) \leq 1,
\end{aligned}
$$

which readily implies that

$$
\lambda_{1}(M) \leq \frac{\|\nabla(f-\bar{f})\|_{2}^{2}}{\|f-\bar{f}\|_{2}} \leq n\left(1+C(p, n)\left(\eta+(d-\pi)_{-}\right)\right),
$$

where we have set $\bar{f}=\frac{1}{\operatorname{Vol} M} \int_{M} f$. 
Remark 3.3. The same technique as in [5] can be used to prove that manifolds with almost positive Ricci curvature and $\lambda_{1}$ is close to $n$ have a diameter close to $\pi$ (see [12]).

\section{Proof of Theorem 1.9}

4.1. Fiber bundle $E$. Let $E$ be the fiber bundle $T M \oplus \mathbb{R} e \rightarrow M$ endowed with the following scalar product and linear connection:

$$
\begin{gathered}
\langle X+f e, Y+h e\rangle_{E}=g(X, Y)+f h, \\
D_{Z}^{E}(X+f e)=D_{Z}^{M} X+f Z+(d f(Z)-g(Z, X)) \cdot e,
\end{gathered}
$$

where $D^{M}$ is the Levi-Civita connection of the metric $g$ on $M$. We denote by $p$ the orthogonal projection of $E$ on $T M, \operatorname{Ric}^{\prime}(S)=\operatorname{Ric}_{M}(p(S))-(n-1) p(S)$ and $\triangle_{\mathrm{sph}}=\bar{\triangle}^{E}+\mathrm{Ric}^{\prime}$.

The following lemma is proved in [3]:

Lemma 4.1. If $f: M \rightarrow \mathbb{R}$ satisfies $\Delta f=\lambda f$, then $S_{f}=\nabla f+f e$ satisfies $\triangle_{\mathrm{sph}}\left(S_{f}\right)=(\lambda-n)(\nabla f-f e)$ and $\left\langle D_{X}^{E} S_{f}, X\right\rangle=D d f(X, X)+f g(X, X)$.

Note also that we have

$$
\mathrm{R}_{(Z, Y)}^{E}(X+f e)=\mathrm{R}^{M}(Z, Y) X-(g(Y, X) Z-g(Z, X) Y) .
$$

4.2. Bound on the Hessian of the first eigenfunction. To prove Theorem 1.9 we need an $L^{\infty}$ bound on the Hessian of the first eigenfunction. For that purpose, we will modify the proof of Theorem 2.4 in [2] (whose proof would give us only a bound on $\left\|D S_{f}\right\|_{n+\epsilon} /\left\|S_{f}\right\|_{\infty}$ for a given $\left.\epsilon=\epsilon(p, n)\right)$. In our case we really need to perform a Moser iteration.

Proposition 4.2. Let $n \geq 2$ and $\infty \geq p>n / 2$. There exists a constant $C(p, n)$ such that if $\left(M^{n}, g\right)$ is any manifold with $\bar{\rho}_{p} \leq \frac{1}{C(p, n)}$ and $\lambda_{1} \leq n+\frac{1}{C(p, n)}$, then for $f: M \rightarrow \mathrm{R}$ such that $\triangle f=\lambda_{1} f$ we have

$$
\frac{\left\|D^{E} S_{f}\right\|_{\infty}}{\left\|S_{f}\right\|_{\infty}} \leq C(p, n)\left(\lambda_{1}+\|\mathrm{R}\|_{2 p}\right)^{\gamma}\left(\left|\lambda_{1}-n\right|+\bar{\rho}_{p}\right)^{\frac{1}{2(1+\gamma)}},
$$

where $S_{f}=\nabla f+f \cdot e$ and $\gamma=\frac{p n}{2 p-n}$.

To prove Proposition 4.2 we need a commutation lemma (see [2]): 
Lemma 4.3. For any section $S \in \Gamma(E)$ we have

$$
\begin{aligned}
\frac{1}{2} \Delta & \left(|D S|^{2}\right)+\left|D^{2} S\right|^{2} \\
& \leq\left\langle D^{*} \mathrm{R}^{E} S, D S\right\rangle+\underline{\mathrm{Ric}}^{-}|D S|^{2}+\langle D \bar{\triangle} S, D S\rangle+\left\|\mathrm{R}^{E}\right\| \cdot|D S|^{2},
\end{aligned}
$$

where $\left\|\mathrm{R}^{E}\right\|$ is the norm of the linear map $\mathrm{R}^{E}: \bigwedge^{2} T_{m} M \rightarrow \bigwedge^{2} E_{m}^{*}$ defined by $\mathrm{R}^{E}(u \wedge v)(T, S)=\left\langle\mathrm{R}^{E}(u, v) T, S\right\rangle$.

Remark 4.4. This lemma is valid for any Riemannian fiber bundle $(E, D,\langle\cdot, \cdot\rangle)$.

We now give the proof of Proposition 4.2.

Proof of Proposition 4.2. We set $u=\sqrt{|D S|^{2}+\epsilon^{2}}$. We have

$$
\begin{aligned}
u \Delta u & =\frac{1}{2} \triangle\left(u^{2}\right)+|d u|^{2}=\frac{1}{2} \triangle\left(u^{2}\right)+\frac{\left|\left\langle D^{2} S, D S\right\rangle\right|^{2}}{|D S|^{2}+\epsilon^{2}} \\
& \leq \frac{1}{2} \triangle\left(|D S|^{2}\right)+\left|D^{2} S\right|^{2} .
\end{aligned}
$$

Hence, by Lemma 4.3

$$
\begin{aligned}
\int_{M}\left|d\left(u^{k}\right)\right|^{2} \leq & \frac{k^{2}}{2 k-1} \int_{M}\left(\frac{1}{2} \Delta|D S|^{2}+\left|D^{2} S\right|^{2}\right) u^{2(k-1)} \\
\leq & \frac{k^{2}}{2 k-1}\left(\int_{M} \underline{\operatorname{Ric}}^{-} u^{2 k}+\int_{M}\langle D \bar{\triangle} S, D S\rangle u^{2(k-1)}\right. \\
& \left.\quad+\int_{M}\left\langle D^{*} \mathrm{R}^{E} S, D S\right\rangle u^{2(k-1)}+\int_{M}\left\|\mathrm{R}^{E}\right\| u^{2 k}\right) .
\end{aligned}
$$

We now apply the divergence theorem to the form $u^{2(k-1)}\left\langle\bar{\triangle} S, D_{\bullet} S\right\rangle$, and get for any $k \geq 1$ :

$$
\begin{aligned}
\int_{M}\langle & D \bar{\triangle} S, D S\rangle u^{2(k-1)} \\
& =\int_{M}|\bar{\triangle} S|^{2} u^{2(k-1)}-2(k-1) \sum_{i} \int_{M}\langle\bar{\triangle} S, D S(i)\rangle d u(i) \cdot u^{2 k-3} \\
& \leq \int_{M}|\bar{\triangle} S|^{2} u^{2(k-1)}+2(k-1) \int_{M}|\bar{\triangle} S||d u| u^{2(k-1)} \\
& \leq \frac{k-1}{2} \int_{M}|d u|^{2} u^{2(k-1)}+(2 k-1) \int_{M}|\bar{\triangle} S|^{2} u^{2(k-1)}
\end{aligned}
$$


We do the same with the form $u^{2(k-1)}\left(\operatorname{tr}_{1,3}\left(\left\langle\mathrm{R}_{(\bullet, \bullet)}^{E} S, D \bullet S\right\rangle\right)\right)$ and get

$$
\begin{aligned}
\int_{M}\left\langle D^{*} \mathrm{R}^{E} S, D S\right\rangle u^{2(k-1)} \\
\quad=\int_{M} \frac{1}{2}\left|\mathrm{R}^{E} S\right|^{2} u^{2(k-1)}+2(k-1) \sum_{i, j} \int_{M}\left\langle\mathrm{R}^{E}(i, j) S, D_{j} S\right\rangle d u(i) u^{2 k-3} \\
\quad \leq \frac{k-1}{2} \int_{M}|d u|^{2} u^{2(k-1)}+(2 k-1) \int_{M}\left|\mathrm{R}^{E} S\right|^{2} u^{2(k-1)}
\end{aligned}
$$

where we have used $\sum_{i, j}\left\langle\mathrm{R}^{E} S(i, j), D^{2} S(i, j)\right\rangle=\frac{1}{2}\left|\mathrm{R}^{E} S\right|^{2}$. $k \geq 1$,

Since $\int_{M}|d u|^{2} u^{2(k-1)}=\frac{1}{k^{2}} \int_{M}\left|d\left(u^{k}\right)\right|^{2}$, the three last inequalities give, for any

$$
\begin{aligned}
\left\|d\left(u^{k}\right)\right\|_{2}^{2} \leq & k\left(\int_{M}{\underline{\mathrm{Ric}^{-}}}^{2 k}+\int_{M}\left\|\mathrm{R}^{E}\right\| u^{2 k}\right) \\
& +k(2 k-1)\left(\int_{M}\left\|\mathrm{R}^{E} S\right\|^{2} u^{2 k-2}+\int_{M}\|\bar{\triangle} S\|^{2} u^{2 k-2}\right) \\
\leq & 4 k^{2}\left(B_{1}\|u\|_{\frac{2 k p}{p-1}}^{2 k}+B_{2}\|S\|_{\infty}^{2}\|u\|_{\frac{2(k-1) p}{p-1}}^{2(k-1)}\right),
\end{aligned}
$$

where we have set

$$
\begin{aligned}
B_{1} & =\left\|{\underline{\mathrm{Ric}^{-}}}^{-}+\right\| \mathrm{R}^{E} \|_{p} \leq C(n)\left(\left\|\mathrm{R}^{M}\right\|_{2 p}^{2}+\lambda_{1}^{2}\right)=B^{2}, \\
B_{2} & =\frac{\|\bar{\Delta} S\|_{2 p}^{2}}{\|S\|_{\infty}^{2}}+\frac{\left\|\mathrm{R}^{E} S\right\|_{2 p}^{2}}{\|S\|_{\infty}^{2}} \leq \frac{\left\|\triangle_{\mathrm{sph}} S\right\|_{2 p}^{2}}{\|S\|_{\infty}^{2}}+\left\|\mathrm{Ric}^{\prime}\right\|_{2 p}^{2}+\left\|\mathrm{R}^{E}\right\|_{2 p}^{2} \\
& \leq C(n)\left(\lambda_{1}^{2}+\left\|\mathrm{R}^{M}\right\|_{2 p}^{2}\right)=B^{2} .
\end{aligned}
$$

By the Sobolev inequality given by Proposition 2.1 we get

$$
\|D S\|_{\frac{2 k n}{n-2}}^{k} \leq\|D S\|_{2 k}^{k}+C(p, n) B k \sqrt{\|D S\|_{\frac{2 k p}{p-1}}^{2 k}+\|S\|_{\infty}^{2} \| D S \frac{\|_{\frac{2(k-1) p}{p-1}}^{2(k-1)}}{2}}
$$

and by $\|D S\|_{2 k} \leq\|D S\|_{\frac{2 k p}{p-1}} \leq\|D S\|_{\infty}^{1 / k}\|D S\|_{\frac{2(k-1) p}{p-1}}^{(1-1 / k)}$ we have

$$
\begin{aligned}
& \left(\frac{\|D S\|_{\frac{2 k n}{n-2}}}{\|D S\|_{\infty}}\right)^{\frac{2 k n}{n-2}} \\
& \quad \leq\left[1+B k C(p, n)\left(1+\frac{\|S\|_{\infty}^{2}}{\|D S\|_{\infty}^{2}}\right)\right]^{\frac{2 n}{n-2}}\left(\frac{\|D S\|_{\frac{2(k-1) p}{p-1}}}{\|D S\|_{\infty}}\right)^{\frac{2(k-1) p}{p-1}}
\end{aligned}
$$


where $v=\frac{n(p-1)}{2 p(n-2)}>1$. We set $k=\frac{a_{n}(p-1)}{2 p}+1$, where $\left(a_{n}\right)_{n}$ is the sequence defined by $a_{0}=\frac{2 p}{p-1}$ and $a_{n+1}=v a_{n}+\frac{2 n}{n-2}$. Then we get

$$
\begin{aligned}
& \left(\frac{\|D S\|_{a_{n+1}}}{\|D S\|_{\infty}}\right)^{\frac{a_{n+1}}{v^{n+1}}} \\
& \quad \leq\left[1+a_{n} C(p, n) B\left(1+\frac{\|S\|_{\infty}^{2}}{\|D S\|_{\infty}^{2}}\right)\right]^{\frac{2 n}{(n-2) v^{n+1}}}\left(\frac{\|D S\|_{a_{n}}}{\|D S\|_{\infty}}\right)^{\frac{a_{n}}{v^{n}}} .
\end{aligned}
$$

Hence

$$
\begin{aligned}
1 & =\lim _{n \rightarrow+\infty}\left(\frac{\|D S\|_{a_{n}}}{\|D S\|_{\infty}}\right)^{\frac{a_{n}}{\nu^{n}}} \\
& \leq \prod_{i=1}^{\infty}\left(1+C(p, n) a_{i} B\left(1+\frac{\|S\|_{\infty}^{2}}{\|D S\|_{\infty}^{2}}\right)\right)^{\frac{2 n}{(n-2) v^{i}}}\left(\frac{\|D S\|_{a_{0}}}{\|D S\|_{\infty}}\right)^{a_{0}} .
\end{aligned}
$$

The Hölder inequality $\|D S\|_{a_{0}} \leq\|D S\|_{2}^{1-\frac{1}{p}}\|D S\|_{\infty}^{\frac{1}{p}}$, gives

$$
\|D S\|_{\infty} \leq \prod_{i=1}^{\infty}\left(1+C(p, n) a_{i} B\left(1+\frac{\|S\|_{\infty}^{2}}{\|D S\|_{\infty}^{2}}\right)\right)^{\frac{n}{(n-2) v^{i}}}\|D S\|_{2} .
$$

If $\|D S\|_{\infty} \geq\|S\|_{\infty}$, then inequality $(*)$ gives

$$
\begin{aligned}
\|D S\|_{\infty} & \leq \prod_{i=1}^{\infty}\left(1+C(p, n) a_{i} B\right)^{\frac{n}{(n-2) \nu^{i}}}\|D S\|_{2} \\
& \leq C(p, n)\left(\lambda_{1}+\|\mathrm{R}\|_{2 p}\right)^{\frac{p n}{2 p-n}}\|D S\|_{2} .
\end{aligned}
$$

If $\|D S\|_{\infty} \leq\|S\|_{\infty}$, then inequality $(*)$ gives

$$
\frac{\|D S\|_{\infty}}{\|D S\|_{2}} \leq\left(\frac{\|S\|_{\infty}}{\|D S\|_{\infty}}\right)^{\frac{2 p n}{2 p-n}} \prod_{i=1}^{\infty}\left(1+C(p, n) a_{i} B\right)^{\frac{n}{(n-2) v^{i}}}
$$

hence

$$
\frac{\|D S\|_{\infty}}{\|S\|_{\infty}} \leq C(p, n)\left(\lambda_{1}+\|\mathrm{R}\|_{2 p}\right)^{\frac{p n}{2 p-n)}}\left(\frac{\|D S\|_{2}}{\|S\|_{\infty}}\right)^{\frac{2 p-n}{2 p-n+2 p n}} .
$$

At this stage note that, by Lemma 4.1 we have

$$
\begin{aligned}
\|D S\|_{2}^{2} & =\left\langle\bar{\triangle}_{\mathrm{sph}}(S), S\right\rangle_{L^{2}}-\left\langle\operatorname{Ric}^{\prime}(S), S\right\rangle_{L^{2}} \\
& \leq\left|\lambda_{1}-n\right|\|S\|_{2}^{2}+\int_{M} \frac{(\underline{\operatorname{Ric}}-(n-1))^{-}}{\operatorname{Vol} M}|S|^{2} \leq\left(\left|\lambda_{1}-n\right|+\bar{\rho}_{p}\right)\|S\|_{\infty}^{2} .
\end{aligned}
$$

Since we have $\frac{2 p-n}{2 p-n+2 p n} \leq 1$, we get the result. 
4.3. Critical points of the first eigenfunction. By Proposition 4.2, the section $S_{f}=\nabla f+f e$ of $E$ satisfies $\left\|D^{E} S_{f}\right\|_{\infty} \leq C(p, n, A)\left(\left|\lambda_{1}-n\right|+\bar{\rho}_{p}\right)^{\frac{1}{1+\gamma}}\left\|S_{f}\right\|_{\infty}$. Since we can suppose the pinching on $\left|\lambda_{1}-n\right|$ and $\bar{\rho}_{p}$ small enough to have

$$
C(p, n, A)\left(\left|\lambda_{1}-n\right|+\bar{\rho}_{p}\right)^{\frac{1}{1+\gamma}} \leq 1 / 4,
$$

the previous inequality and Theorem 1.6 give

$$
\begin{aligned}
\inf \left|S_{f}\right| & \geq\left[1-C(p, n, A)\left(\left|\lambda_{1}-n\right|+\bar{\rho}_{p}\right)^{\frac{1}{1+\nu}}\right]\left\|S_{f}\right\|_{\infty} \\
& >C(p, n, A)\left(\left|\lambda_{1}-n\right|+\bar{\rho}_{p}\right)^{\frac{1}{1+\nu}}\left\|S_{f}\right\|_{\infty} \geq\left\|D^{E} S_{f}\right\|_{\infty} .
\end{aligned}
$$

We infer that if $x_{0}$ is a critical point of $f$, then by Lemma 4.1 we have

$$
\left|D d f_{x_{0}}(X, X)+f\left(x_{0}\right)\right|=\left|\left\langle D_{X}^{E} S_{f}, X\right\rangle_{E}\right| \leq\left\|D^{E} S_{f}\right\|_{\infty}<\left|S_{f}\left(x_{0}\right)\right|=\left|f\left(x_{0}\right)\right|
$$

for any unit vector $X$ of $T_{x_{0}} M$. Hence we have $-\left|f\left(x_{0}\right)\right|-f\left(x_{0}\right)<D d f_{x_{0}}(X, X)<$ $\left|f\left(x_{0}\right)\right|-f\left(x_{0}\right)$ for any critical point $x_{0}$ of $f$. So the only critical points of $f$ are non degenerate global extrema, which implies that $M$ is homeomorphic to $\mathbb{S}^{n}$ by Reeb's theorem.

\section{References}

[1] M. Anderson, Metrics of positive Ricci curvature with large diameter. Manuscripta Math. 68 (1990), 405-415. Zbl 0711.53036 MR 1068264

[2] E. Aubry, B. Colbois, P. Ghanaat, E. Ruh, Curvature, Harnack's inequality, and a spectral characterization of nilmanifolds. Ann. Glob. Anal. Geom. 23 (2003), 227-246. Zbl 1026.58021 MR 1966846

[3] E. Aubry, Pincement sur le spectre et le volume en courbure de Ricci positive. Ann. Sci. École Norm. Sup. 38 (2005) 387-405. Zbl 1085.53024 MR 2166339

[4] E. Aubry, Finiteness of $\pi_{1}$ and geometric inequalities in almost positive Ricci curvature. Ann. Sci. École Norm. Sup. 40 (2007), 675-695. Zbl 1141.53034

[5] P. Bérard, G. Besson, S. Gallot, Sur une inégalité isopérimétrique qui généralise celle de Paul Levy-Gromov. Invent. Math. 80 (1985), 295-308. Zbl 0571.53027 MR 0788412

[6] S. Cheng, Eigenvalue comparison theorems and its geometric applications. Math. Z. 143 (1975), 289-297. Zbl 0329.53035 MR 0378001

[7] C. Croke, An eigenvalue pinching theorem. Invent. Math. 68 (1982), 253-256. Zbl 0505.53018 MR 0666162

[8] S. Ilias, Un nouveau résultat de pincement de la première valeur propre du laplacien et preuve de la conjecture du diamètre pincé. Ann. Inst. Fourier 43 (1993), 843-863. Zbl 0783.53024 MR 1242618 
[9] M. Le Couturier, G. Robert, $L^{p}$-pinching and the geometry of compact Riemannian manifolds. Comment. Math. Helv. 69 (1994), 249-271. Zbl 0821.53033 MR 1282370

[10] Y. Otsu, On manifolds of positive Ricci curvature with large diameter. Math. Z. 206 (1991), 255-264. Zbl 0697.53042 MR 1091941

[11] G. Perelman, A diameter sphere theorem for manifolds of positive Ricci curvature. Math. Z. 218 (1998), 595-596. Zbl 0831.53033 MR 1326988

[12] P. Petersen, C. Sprouse, Integral curvature bounds, distance estimates and applications. $J$. Differential Geom. 50 (1998), 269-298. Zbl 0969.53017 MR 1684981

[13] T. Sakai, Riemannian Geometry. Transl. Math. Monogr. 149, Amer. Math. Soc., Providence, RI, 1996. Zbl 0886.53002 MR 1390760

Received March 26, 2007

Erwann Aubry, Laboratoire Dieudonné, Université Nice Sophia-Antipolis, Parc Valrose, 06108 Nice, France

E-mail: eaubry@math.unice.fr 